\title{
Mission d'étude du programme ANR « Géologie et architecture à Délos » - 2018
}

Rapport 2018

Jean-Charles Moretti

\section{(2) OpenEdition \\ Journals}

Édition électronique

URL : http://journals.openedition.org/baefe/1738

DOI : $10.4000 /$ baefe. 1738

ISSN : 2732-687X

Éditeur

ResEFE

Référence électronique

Jean-Charles Moretti, « Mission d'étude du programme ANR « Géologie et architecture à Délos »-

2018 » [notice archéologique], Bulletin archéologique des Écoles françaises à l'étranger [En ligne], Grèce, mis en ligne le 01 novembre 2020, consulté le 22 mars 2021. URL : http://journals.openedition.org/ baefe/1738; DOI : https://doi.org/10.4000/baefe.1738

Ce document a été généré automatiquement le 22 mars 2021.

\section{(c) (i) (3)}

Le Bulletin archéologique des Écoles françaises à l'étranger est mise à disposition selon les termes de la Licence Creative Commons Attribution - Pas d'Utilisation Commerciale - Pas de Modification 4.0

International. 


\section{Mission d'étude du programme ANR « Géologie et architecture à Délos » - 2018}

Rapport 2018

Jean-Charles Moretti

\section{NOTE DE L'AUTEUR}

Autorité nationale présente : Éphorie des Cyclades

Numéro de mission : C56

Composition de l'équipe de terrain : Jean-Charles Moretti (CNRS, IRAA), Emmanuel Desroches (restaurateur), Lionel Fadin, (topographe EFA), Pavlos Karvonis (Académie des sciences d'Athènes), Isabelle Moretti (géologue), Catherine Raad (étudiante stagiaire), Violaine Sautter (CNRS, MNHN), Océan Tricquet (topographe stagiaire ESGT), Stéphanie Zugmeyer (architecte, AMU, IRAA).

Établissements porteurs de l'opération : EFA

Établissements porteurs de l'opération : EFA

\section{Données scientifiques produites :}

Carnet Hypothèses

1 Cette deuxième mission a permis de finaliser la liste des faciès et sous-faciès de roches existants à Délos dans les carrières comme dans le bâti antique, d'établir les bases d'une première quantification des volumes extraits dans les 37 zones d'extraction identifiées en $2017^{1}$ et de commencer à relever certaines d'entre elles et les traces d'exploitation les plus caractéristiques qui s'y trouvent.

2 Nous avons constitué une lithothèque de Délos détaillant les différents faciès au sein des quatre grands groupes de roches (granite, gneiss, marbre et poros) présents dans le substrat géologique comme dans le bâti. Des amphibolites vert sombre, interstratifiées 
à des pyroxénites vert clair et associées au marbre, sont les seules roches basiques dans l'île. Une fiche a été établie pour chaque faciès.

3 Les granites présentent quatre sous-groupes : à amphibole ; à mica ; hyper-quartzeux ; hyper-quartzeux à grains fins et niveaux riches en grenat ou en tourmaline dans le Sud de l'île ; aplite à grains fin pauvre en mica recoupant les gneiss dans le Nord de l'île.

4 Les gneiss se déclinent en quatre faciès : des gneiss gris foncé rubanés formant les socles du Nord de l'île; des gneiss micacés avec une patine rouille caractéristique, présents en lentille dans les granites; des gneiss beige clair dans le Sud-Ouest de l'île. Il existe enfin une variété de gneiss argenté à mica blanc d'aspect gris clair très fréquente dans le bâti, mais dont on n'a pas trouvé a trace dans la géologie de l'île.

5 Le marbre aussi se décline en quatre faciès : saccharoïdes équigranulaires (grain de 3 à $4 \mathrm{~mm}$ ) avec à grande échelle des passées bleutées (C24 sur Ghlastropi); fin équigranulaire saccharoïde montrant une alternance de niveaux ocre-jaune et grisâtre (C23 dans Colline du théâtre) ; blanc cassé jaune et blanc et très fracturé, sans doute pas exploité, au sud-ouest de l'île, à l'Ouest de la branche Sud de la Terrasse en gamma (C32) ; hétéro granulaire à cristaux pluri-centimétrique de calcite bleuté, très maclés (C25 au Sud-Est du Cynthe).

6 Le "poros » est présent dans les constructions sous trois faciès, un faciès éolien, des caliches (paléosols) et des beachrocks (diagénèse précoce de carbonate marquant les paléo rivages). Quelques affleurements existent, mais aucun ne peut réellement être identifié comme carrière. Les seuls beachrocks connus en place sont sous l'eau car le niveau relatif de la mer a monté de plus de $2,5 \mathrm{~m}$ depuis $2400 \mathrm{ans}^{2}$.

7 Pour chacune des 37 zones d'extraction, une fiche a été élaborée regroupant toutes les données de base (coordonnées, surface exploitée, description géologique de la ou des roches extraites, liste des types de traces d'exploitation, photographies, volume extrait estimé). Il est à noter que si toutes les carrières de granite et de marbre (C34 mis à part) conservent des traces d'extraction (saignées, encoignures, emboîtures) et certaines des blocs bruts ou équarris abandonnés sur place $(\mathrm{C} 26,28$ et 31 pour se limiter aux carrières antiques), celles de gneiss ne sont souvent identifiables que par leur géométrie "non naturelle ». La facilité de clivage, en bénéficiant des fractures en vertical et de la schistosité à l'horizontal, a sans doute joué un rôle dans ces différences. Il apparaît enfin que toutes les grandes carrières sont situées soit dans l'agglomération antique, soit au bord de la mer, à proximité d'une zone d'embarquement potentielle. Seules les petites carrières, et en particulier les carrières en creux du nord, semblent avoir été exploitées par transport terrestre. Il semble que dans l'Antiquité, l'extraction et parfois même l'ouverture des carrières aient été réalisées à la demande pour répondre à des commandes. Il n'y a pas eu d'extraction continue avec stockage des blocs en vue de leur vente.

8 Nous avons entrepris une première approche des techniques d'extraction antiques et modernes par l'identification et l'analyse des traces d'extraction non abouties et des objets inachevés conservés dans les carrières. Pour certaines d'entre elles, nous avons commencé à réaliser des plans et des profils d'ensemble, à relever des traces significatives et à rédiger une description détaillée qui rende compte du mode d'exploitation. À titre d'exemple nous donnons pour la carrière C29 une orthophographie (fig. 1) et un relevé de saignées et d'emboîtures sur des fronts de taille (fig. 2). Des profils ont été réalisés dans les carrières $C 10,11,13,21,28$ et 29 ; des photogrammétries par drone dans $\mathrm{C} 25,28,31$ et 34 ; des relevés de détails dans $\mathrm{C} 28$ 
et 29, ainsi que dans l'Asclépieion, où sont conservés des encoignures ainsi que les vestiges d'un outil en fer : coin ou paumelle (fig. 3).

Fig. 1. La carrière C29 : orthophotographie.

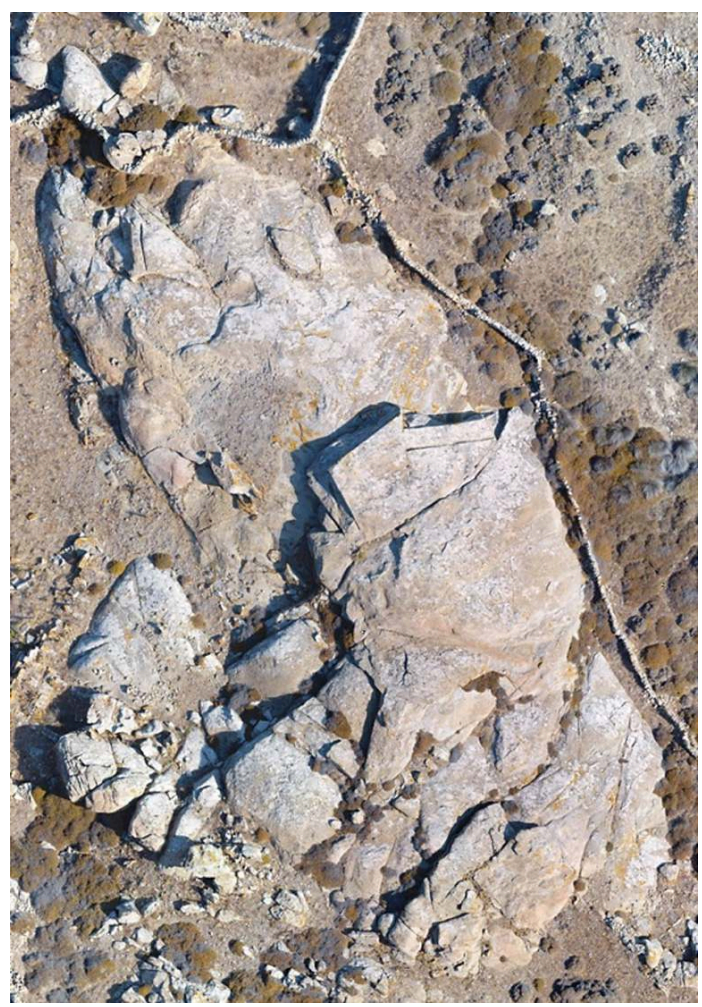

GAD / EFA, L. Fadin, EFA.

Fig. 2. La carrière C29 : relevé de saignées et d'emboîtures sur les fronts de taille au 1/25.

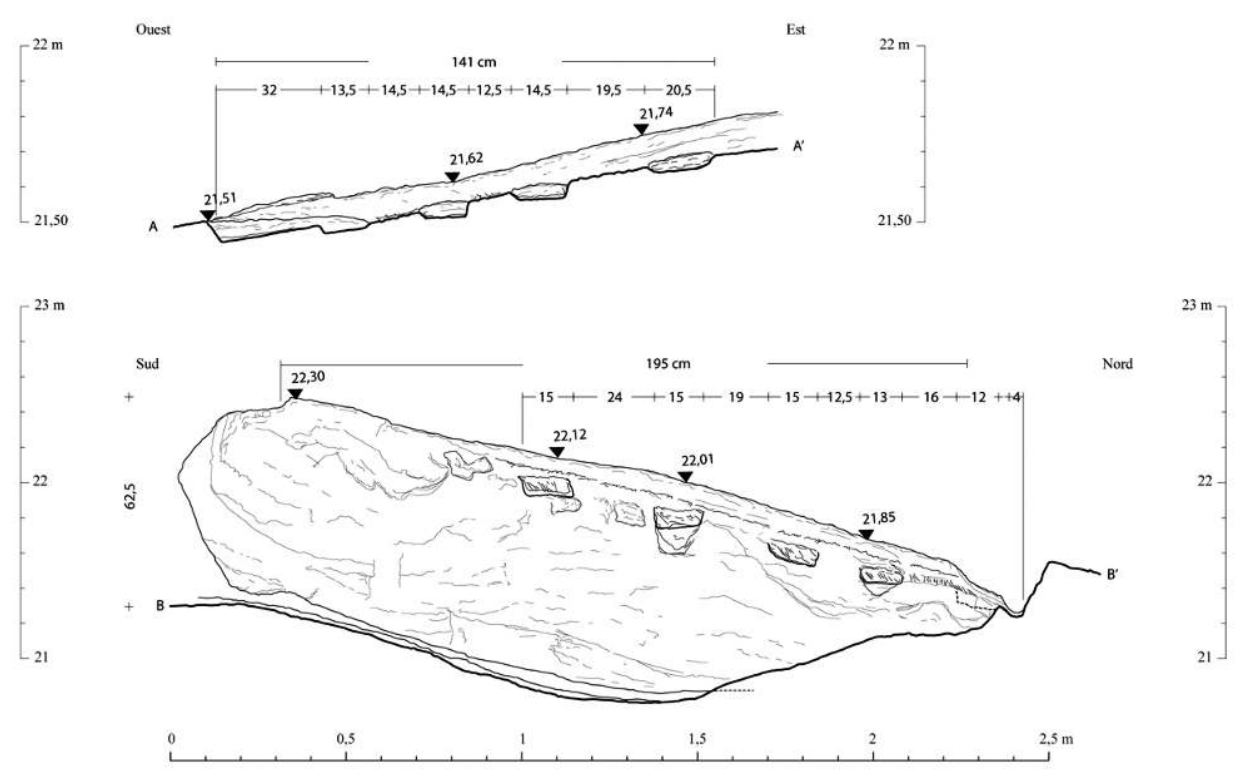

GAD / EFA, C. Raad. 
Fig. 3. Encoignures dans le Péristyle de l'Asclépieion : plan et profils au 1/10 et 1/5.

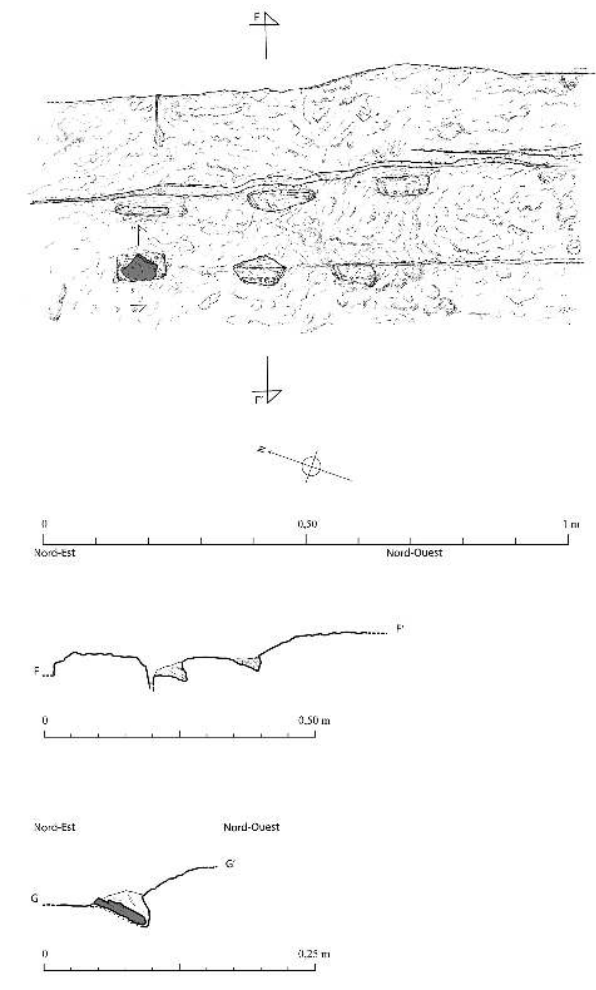

GAD / EFA, C. Raad.

9 La volumétrie rapide de l'ensemble des marbres et des roches granitiques et gneissiques, confrontée à la volumétrie non moins rapide des constructions antique permet de faire le constat suivant. Les granites constitueraient près de $50 \%$ du volume extrait ce qui devrait suffire au volume de roches mises en œuvre dans l'île. La quantité de gneiss est limitée, environ $30 \%$ du volume extrait, alors qu'il est largement majoritaire dans le bâti. Il a donc nécessairement été importé à grande échelle. La plus grande exploitation de marbre, qui est située au centre du site, entre le théâtre et le barrage de l'Inopos (C23), montre une alternance des marbres avec des amphibolites et de gneiss marrons. Si l'exploitation massive du marbre pour la construction du théâtre ne fait aucun doute, comme celle des amphibolites repérables dans les maisons attenantes à la carrière (îlot de la maison de l'Inopos), celle du gneiss marron reste à cartographier précisément dans le Quartier du théâtre d'une part et dans les constructions situées entre le barrage de l'Inopos et le Sanctuaire de la déesse syrienne de l'autre.

Les questions à résoudre les années à venir en termes de provenance concernent les gneiss argentés, les marbres et les poros. En 1911, L. Cayeux avait suggéré que ces gneiss à muscovite argenté, qui semblent absents dans les formations géologiques de Délos, puissent être importés de l'île voisine de Rhénée, ce qui reste à confirmer. Une rapide reconnaissance dans les Rhevmatiaris a permis de noter la présence de certains sites d'extraction du gneiss, mais rien de significatif pour combler le volume estimé manquant. Certains marbres sont clairement importés et la détermination précise de leur origine, en particulier en comparaison avec des échantillons de marbre des autres îles des Cyclades (Tinos, Paros, Naxos...) et de la région d'Athènes débute au Museum national d'histoire naturelle. Les origines possibles des différents types de poros, en 
particulier par des prospections à Rhénée et à Mykonos où de nombreux affleurements ont été signalés, restent à préciser. Une volumétrie précise de chacune des roches dans le bâti reste aussi à faire.

\section{BIBLIOGRAPHIE}

EAD XLIV

Roland Étienne (éd.), Le sanctuaire d'Apollon à Dëolos, 1 : Architecture, topographie, histoire, Athènes, EFA, EAD 44, 2018.

\section{NOTES}

1. Pour leur emplacement et leur numérotation, voir la fig. 1 de la chronique de 2017.

2. En dernier lieu : St. DESRUELLES, Cl. HASENOHR, dans EAD XLIV, p. 41.

\section{INDEX}

Thèmes : EFA

sujets https://ark.frantiq.fr/ark:/26678/pcrts8SiTTY3Ka, https://ark.frantiq.fr/ark:/26678/ pcrtXk6sdvTjnE, https://ark.frantiq.fr/ark:/26678/pcrtxzTYzVBKas, https://ark.frantiq.fr/ark:/ 26678/pcrtSkipOsBGML, https://ark.frantiq.fr/ark:/26678/pcrtDnenSUMjFl

lieux https://ark.frantiq.fr/ark:/26678/pcrtXvsaqTQa4J, https://ark.frantiq.fr/ark:/26678/ pcrtKgel43EsfJ

chronologie https://ark.frantiq.fr/ark:/26678/pcrtNzYn31IIAZ

Année de l'opération : 2018

\section{AUTEURS}

\section{JEAN-CHARLES MORETTI}

Institut de recherche sur l'architecture antique, CNRS, Maison de l'Orient et de la Méditerranée 